CHAPTER 18

\title{
Diamond-based Resonators for Chemical Detection
}

\author{
EMMANUEL SCORSONE* AND ADELINE TROUVÉ
}

CEA-LIST, Diamond Sensors Laboratory, Gif-sur-Yvette 91191, France

${ }^{*}$ Email: emmanuel.scorsone@cea.fr

\subsection{Introduction}

Chemical/biochemical sensors are devices that transform chemical or biological information into an analytically useful signal. Generally speaking, they are the result of coupling a selective layer to a physical part known as the transducer. The sensors may be classified according to the operating principle of the transducer. In brief, the main transduction phenomena are optical, electrochemical, electrical and gravimetric. ${ }^{1}$ A wide variety of innovative chemical and biochemical sensor technologies are being reported in the literature every year, often showing highly promising performances. However, when it comes to commercial sensors, one has to admit that the market is very conservative. A possible explanation for this is the lack of robustness and reliability of the newly developed sensors and, therefore, their difficulty to maintain performance specifications under adverse operating conditions. ${ }^{2}$

The robustness of the sensors is linked, on the one hand, to their ability to withstand mechanical shocks, stresses, or vibrations. On the other hand, it is related the chemical stability of the selective layer in the operating environment. The variety of exceptional physicochemical properties of diamond materials are generally extremely resilient to chemical degradation. They also feature outstanding mechanical properties, such as a high Young's

RSC Nanoscience \& Nanotechnology No. 31

Nanodiamond

Edited by Oliver Williams

(C) The Royal Society of Chemistry 2014

Published by the Royal Society of Chemistry, www.rsc.org 
modulus and a strong resistance to fracture. Furthermore, diamond is mainly composed of carbon atoms, which can be made available on the material surface for covalent grafting of chemical receptors via strong carbon-carbon bonds. Hence, it becomes obvious that diamond can play an important role toward improving the stability and reliability of chemical or biochemical sensors. Additionally, when looking more closely at the range of remarkable physical and chemical properties of diamond, one can also predict that it is of high interest for improving the transducing characteristics of sensors, such as their sensitivity levels. Let us consider boron-doped diamond electrodes for instance: their wide potential window in aqueous media allows them to address target analytes that could not be measured with standard platinum or other carbon-based electrodes; ${ }^{3}$ also, the low background current, resulting from low electrical double layer capacitance of the electrode, generally gives rise to better signal-to-noise ratios when compared with other electrode materials. ${ }^{4}$ Thus, there has been an increasing interest in diamond materials for the development of chemical sensors, as reflected by the increasing number of scientific publications in this area over the last decade.

Many interesting chemical/biochemical diamond-based sensor architectures have been developed recently that will be summarised in section 18.2. Nevertheless, we have chosen in this chapter to focus on two particular types of transducer technologies: cantilevers and surface acoustic wave (SAW) sensors, which offer a number of significant advantages over other types of transducers. For example, today, biosensors are mainly based on electrochemical and optical transduction methodologies. Electrochemical transduction answers the main requirements associated to real time in situ portable sensors. These capabilities, as well as material costs, which allow the design of one-shot sensors, constitute the basis of their success, for example, towards glucose detection in point-of-care diagnostics. However, even though electrochemistry is well suited to enzymatic biochemical sensors in which redox enzymes are intimately associated to the electrode transducer, it suffers from a lack of accuracy, sensitivity and selectivity in the case of non-metabolistic detection that uses biological receptors, such as antibodies, proteins or DNA. For these latter applications, the sensing principles are mainly based on optical instrumentations (fluorescence microscopy, surface plasmon resonance or colorimetry), which are usually cumbersome and unfit for handheld applications. In contrast, since SAW sensors and cantilevers measure mass (sometimes amongst other parameters), and since all molecules have a mass, they are capable, in principle, of detecting any type of analyte independently, for instance, of their optical or electrochemical properties. These sensors generally offer also the possibility to achieve higher sensitivity performances than with other types of transducers. Finally, they can be fabricated according to the advances of micro- and nano-technologies. As a result, they are compatible with low cost manufacturing and miniaturised systems. Hence they appear highly promising for a wide range of "low cost" portable sensing applications. 
In this context the goal of this chapter is to give some insight into the potential of diamond for the development of high performance chemical sensors. The first section summarises a few examples of sensors and the various exceptional properties of diamond that can be exploited in chemical sensing. The next two sections describe in more detail, for the reasons given above and also because they illustrate well the benefit of diamond for such applications, cantilever and SAW transducer technologies.

\subsection{Diamond Materials: Some Remarkable Properties for the Development of High Performance Chemical Sensors}

\subsubsection{Physical Properties}

Over the last decades, silicon has been used extensively for microelectromechanical systems (MEMS) fabrication, taking advantage of the available silicon-micromachining techniques developed for integrated circuits technology. For example, silicon is used for the fabrication of accelerometers, pressure sensors, resonators and chemical/biochemical sensors. Nevertheless, diamond exhibits superior mechanical properties over silicon, including, for instance, a lower friction coefficient, a higher resistance to fracture and a much higher Young's modulus ${ }^{5-7}$ (Table 18.1). These outstanding mechanical properties of diamond materials are well known. They have been exploited for many years for cutting, polishing or drilling tools. Actually, those assets are also very promising for the development of MEMS, and such devices may be useful for chemical detection.

Table 18.1 Outstanding linear mechanical properties of single-crystal diamond (calculated or measured) and CVD diamond (measured). (Reprinted with permission from ref. 7; copyright (2012) America Institute of Physics.)

\begin{tabular}{lll}
\hline & Single-crystal diamond & CVD diamond \\
\hline Bulk modulus & $433 \mathrm{GPa}$ & $443 \mathrm{GPa}$ \\
Shear modulus & $502 \mathrm{GPa}$ & $507 \mathrm{GPa}$ \\
$\begin{array}{l}\text { Young's modulus, anisotropy } \\
\text { (Random) crystallites }\end{array}$ & $1050-1210 \mathrm{GPa}$ & \\
$\begin{array}{l}\text { Poisson ratio, anisotropy } \\
\text { (Random) crystallites }\end{array}$ & $1143 \mathrm{GPa}$ & $500-1200 \mathrm{GPa}$ \\
Sound velocity, long. (111) & $0.00786-0.115$ & 0.075 \\
Sound velocity, long. (100) & 0.0691 & $18784 \mathrm{~m} \mathrm{~s}^{-1}$ \\
Sound velocity, long. (110) & $19039 \mathrm{~m} \mathrm{~s}^{-1}$ & \\
Rayleigh velocity, (110) texture & $18038 \mathrm{~m} \mathrm{~s}^{-1}$ & $18182 \mathrm{~m} \mathrm{~s}^{-1}$ \\
Rayleigh velocity, polycrystal. & $10753 \mathrm{~m} \mathrm{~s}^{-1}$ & $10326 \mathrm{~m} \mathrm{~s}^{-1}$ \\
Vibrational frequency (Raman) & $10930 \mathrm{~m} \mathrm{~s}^{-1}$ & $10850 \mathrm{~m} \mathrm{~s}^{-1}$ \\
Expansion coefficient & $1332.2 \mathrm{~cm}^{-1}$ & $1332 \mathrm{~cm}^{-1}$ \\
& & $0.8 \times 10^{-6} \mathrm{~K}^{-1}$ \\
& & $0.9 \times 10^{-6} \mathrm{~K}^{-1}$ \\
\hline
\end{tabular}


Various diamond-based MEMS have been investigated and reported in the literature, such as RF switches. ${ }^{8}$ Diamond cantilevers with high Q-factors were also characterised (Figure 18.1). ${ }^{9}$ Some were recently used for chemical detection (section 18.3). Other systems, like film bulk acoustic resonators (FBAR) operating at $3.5 \mathrm{GHz}$, have been reported. ${ }^{10}$ Such devices hold promise too for the development of gravimetric chemical sensors of extreme sensitivity.

Diamond also exhibits some remarkable electronic properties. Doped diamond is a wide gap semiconductor that may reach intrinsic electron and hole mobilities of, typically, $\geq 3100 \mathrm{~cm}^{2} \mathrm{~V}^{-1} \mathrm{~s}^{-1}$ and $\geq 3200 \mathrm{~cm}^{2} \mathrm{~V}^{-1} \mathrm{~s}^{-1}$ at $300 \mathrm{~K}$, respectively. In practice, only p-doping with boron is well controlled and enables such performances. Boron doping in polycrystalline diamond may also be achieved and, although its electronic properties are not as good as that of single crystals, such a material remains very attractive in, for example, electrochemical applications. In contrast with other semiconductors like silicon, boron-doped diamond does not contain a surface oxide layer. Hence, the semiconducting material is available directly to interact with the external medium, which is clearly very advantageous for many chemical sensing applications.

Several types of solid state chemical sensors exploiting the semiconducting properties of diamond have been investigated. For instance, Gurbuz and coworkers demonstrated the performance of catalyst/adsorptive-oxide/ insulator/semiconductor (CAIS) devices based on doped diamond/intrinsic diamond/metal catalyst for the detection of hydrogen, oxygen, carbon monoxide (Figure 18.2) or various hydrocarbons. ${ }^{11}$ The influence of gases used in the semiconductor industry at temperatures typically above

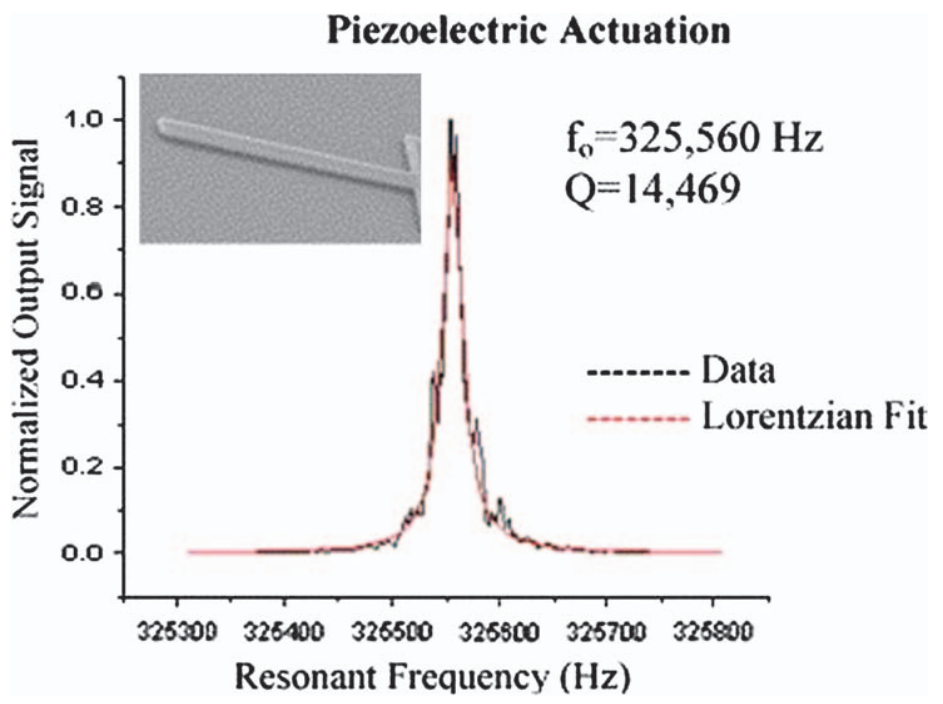

Figure 18.1 Typical response of piezoelectric actuated poly-C cantilever resonator. (Adapted from ref. 9 with permission from Elsevier.) 

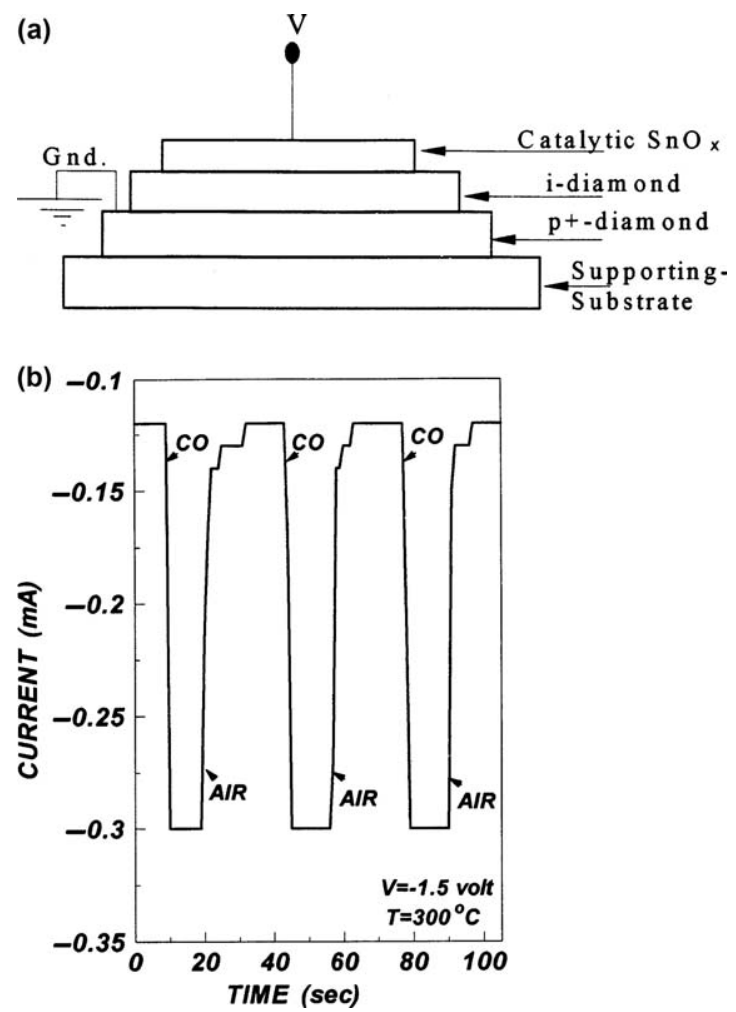

Figure 18.2 a) Diamond-based CAIS sensor, b) typical response of CAIS sensor to 9.4 Torr CO in 0.4 Torr oxygen atmosphere.

(Reprinted from ref. 22 with permission from Elsevier.)

$\geq 100{ }^{\circ} \mathrm{C},{ }^{12,13}$ as well as that of $\mathrm{CO}$ gas at room temperature, ${ }^{14}$ on the resistivity of doped diamond layers was also investigated.

Furthermore, hydrogen-terminated diamond exhibits a p-type surface conductivity due to the presence of an electrolytic layer resulting from adsorption of water molecules and other adsorbates from the environment onto the surface. ${ }^{15}$ This surface conductivity is highly dependent on the $\mathrm{pH}$ in aqueous media when the surface is slightly oxidised, which has led to the development of $\mathrm{pH}$ sensors based on those conductivity phenomena. ${ }^{16}$ Flat $^{17,18}$ or nano-structured ${ }^{19,20}$ hydrogenated diamond surfaces were also studied for gas sensing, where surface conductivity variations are measured upon gas exposure.

The thermal conductivity of diamond single crystal lies in the order of $2000 \mathrm{~W} \mathrm{~m}^{-1} \mathrm{~K}^{-1}$ at $300 \mathrm{~K}$, which is approximately five times higher than copper. This extremely high value has been exploited in the development of high power components or even for silicon-on-diamond (SOD) substrates for heat dissipation applications in microelectronics. ${ }^{21}$ This property of diamond may also be beneficial for designing catalytic gas sensors in which fast 
temperature cycles may be useful, either for energy saving management or because the selectivity of the sensor may be tuned by changing its operating temperature. ${ }^{22}$ In addition, diamond, being a wide bandgap semiconductor, exhibits electronic properties that remain more stable than silicon at high temperatures. Therefore, it may be seen as a good candidate for the design of stable gas sensors operating at temperatures up to $400{ }^{\circ} \mathrm{C}$. Finally, it is interesting to mention that a thermocouple containing a diamond/ palladium junction has been assessed for the detection of hydrogen gas. ${ }^{23}$

Diamond's wide bandgap offers extreme optical transparency from ultraviolet to infrared, together with a high refractive index, in the order of 2.5 at $600 \mathrm{~nm}$. These optical properties, when combined with the chemical properties, are promising for the development of robust and sensitive chemical/ biochemical transducers. For instance, label-free photonic crystal biosensors have recently been shown to be a highly sensitive method for performing a wide variety of biochemical assays. These devices are generally made of silicon. They operate in the infrared spectral region and are somehow limited in the chemistry that can be used to immobilise bio-receptors onto their surface. In contrast, diamond photonic crystals are particularly interesting here because they can potentially operate in the visible region with an extremely high quality factor and small volume cavities, and offer again highly stable carbon chemistry. Recently, nanocrystalline diamond photonic crystals were reported with a Q-factor as high as 2800 and it is hoped in the future to reach Q-factors up to $10000 .^{24,25}$ Therefore, diamond offers a bright future in the area of optical biosensor chips.

\subsubsection{Chemical Properties}

Diamond materials are obviously also very attractive for their chemical properties. First of all, they are very inert and chemically resilient to, for instance, corrosion, at temperatures up to around $400{ }^{\circ} \mathrm{C}$, where they may start to deteriorate when in the presence of oxygen. These properties make it highly stable even in very harsh environments. This is one reason, for instance, why radiation diamond detectors were developed in the past because they could withstand the high temperature, high pressure and highly acidic operating conditions in nuclear power plants. ${ }^{26}$ This high stability of diamond may open new fields of applications for chemical sensing in harsh environments, but it also contributes to the increasing robustness and reliability of chemical sensors in more conventional operating conditions.

Diamond also offers a carbon-terminated surface, which may be made available for grafting a wide range of organic receptors through strong covalent carbon-carbon bonds. Several chemical ${ }^{27,28}$ and electrochemical routes ${ }^{29}$ for surface grafting on diamond have been reported. Such modified surfaces have been used for the development of a wide range of chemical or biochemical sensors. ${ }^{30,31}$

Furthermore, boron-doped diamond features remarkable electrochemical properties. These include a wide potential window that can exceed $3 \mathrm{~V}$ in 


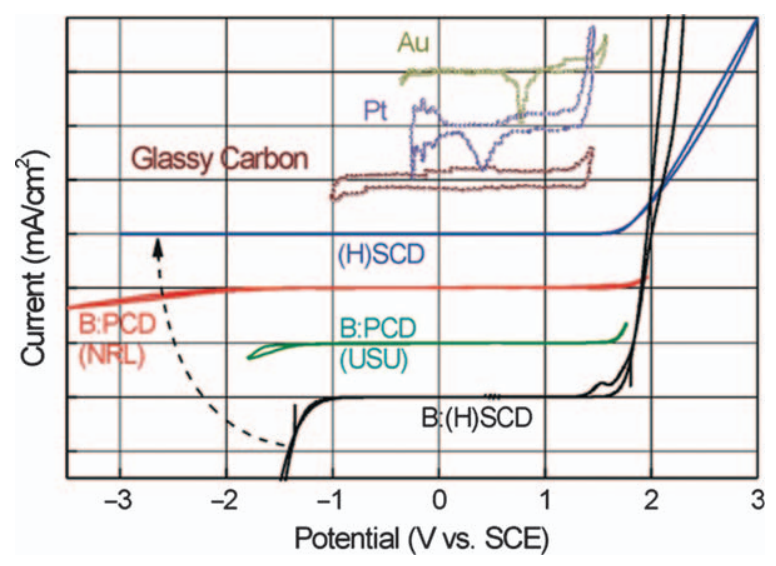

Figure 18.3 Voltammograms for water electrolysis on various electrodes. The supporting electrolyte is $0.5 \mathrm{M} \mathrm{H}_{2} \mathrm{SO}_{4}$. The graphs are shifted vertically for comparison. Two polycrystalline films, B: PCD (provided by the Naval Research Laboratory, NRL) with $5 \times 10^{19} \mathrm{~B} \mathrm{~cm}^{-3}$ and B: PCD (provided by Utah State University, USU) with $5 \times 10^{20} \mathrm{~B} \mathrm{~cm}^{-3}$, are compared with a single crystalline boron-doped diamond, B: (H)SCD (single crystalline diamond) with $3 \times 10^{20} \mathrm{~B} \mathrm{~cm}^{-3}$, and with an undoped diamond (H)SCD. Also shown are data for Pt, Au and glassy carbon. Oxidation reactions, e.g., oxygen evolution, have positive currents and emerge around $1.8 \mathrm{~V}$ for all diamond samples. Reduction reactions, e.g., hydrogen evolution, have negative currents and show very different properties. Note that the background current within the regime between hydrogen and oxygen evolution for diamond is very low and the electrochemical potential window is large compared with glassy carbon, Pt and Au.

(Reprinted from ref. 29 with permission from IOP.)

aqueous media (Figure 18.3) and a low background current, as well as a high reactivity comparable to that of platinum after electrochemical activation. ${ }^{32}$ Moreover, because of the high atomic density of diamond, diffusion of chemical or biological species into the material is impossible; hence, fouling of the electrodes is only limited to physico-chemical adsorption of high molecular weight species at the surface. Thus, the electrodes are generally less prone to fouling. Moreover, it was demonstrated that such bare diamond electrodes may be reactivated after fouling in biological mediums and, therefore, potentially used for in situ continuous monitoring (Figure 18.4). ${ }^{33}$ Thus, BDD electrodes are promising for chemical detection and several applications have been investigated over the years. The wide potential window of diamond enabled the detection of nitroaromatic pollutants in seawater where platinum failed because the reduction potential of the target chemicals falls outside the solvent reduction front of platinum electrodes. ${ }^{3}$ Diamond electrodes have also proven to be efficient for the detection of glucose, ${ }^{34}$ lectins, ${ }^{35}$ DNA, ${ }^{36}$ etc. in the liquid phase, as well as arsine in the gas phase, ${ }^{37}$ to name but a few. 

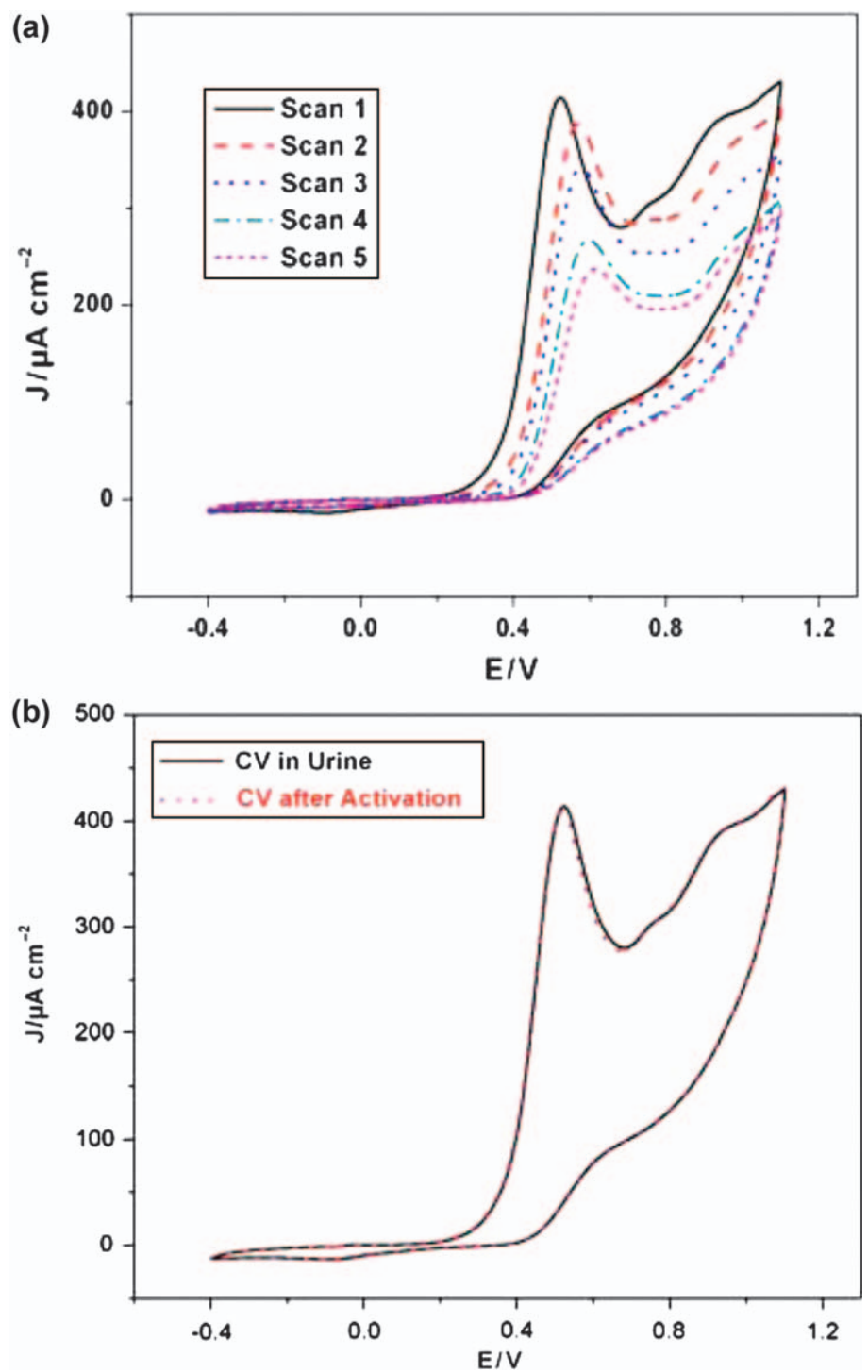

Figure 18.4 a) Cyclic voltammogram in human urine from $-0.4 \mathrm{~V}$ to $1.1 \mathrm{~V} v s$. $\mathrm{Ag} / \mathrm{AgCl}$ at $100 \mathrm{mV} \mathrm{s}^{-1}$ measured with BDD electrode, where $J$ is the current density in $\mu \mathrm{A} \mathrm{cm}^{-2}$ and $E$ is the applied voltage in V. The electrode was cleaned thoroughly in deionised water prior to each scan and, hence, the attenuation of the peak is due to fouling and not because the solution surrounding the electrode is depleted of electro-active species; b) comparison of the cyclic voltammogram of "as-grown" electrode (solid line) and the same electrode after activation (dotted line), where $J$ is the current density in $\mu \mathrm{A} \mathrm{cm}^{-2}$ and $E$ is the applied voltage in V. The electrolyte is human urine and the scan rate is $100 \mathrm{mV} \mathrm{s}^{-1}$.

(Reproduced with permission of the Electrochemical Society from ref. 33.) 
Sometimes, electrochemical measurements may be coupled to simultaneous gravimetric or viscosity monitoring using typically quartz crystal microbalances (QCM) and, quite logically, the fabrication of diamond-QCM has been attempted. Here, the difficulty is related to the lack of compatibility between diamond growth temperature conditions (typically $>600{ }^{\circ} \mathrm{C}$ ) and the phase transition temperature for $\alpha$-quartz $\left(513^{\circ} \mathrm{C}\right)$. Approaches to overcome this issue consist of attaching a previously grown freestanding diamond film onto the $\mathrm{QCM}^{38}$ or to use a high-temperature-stable piezoelectric material, such as langasite ${ }^{39}$ (Figure 18.5).
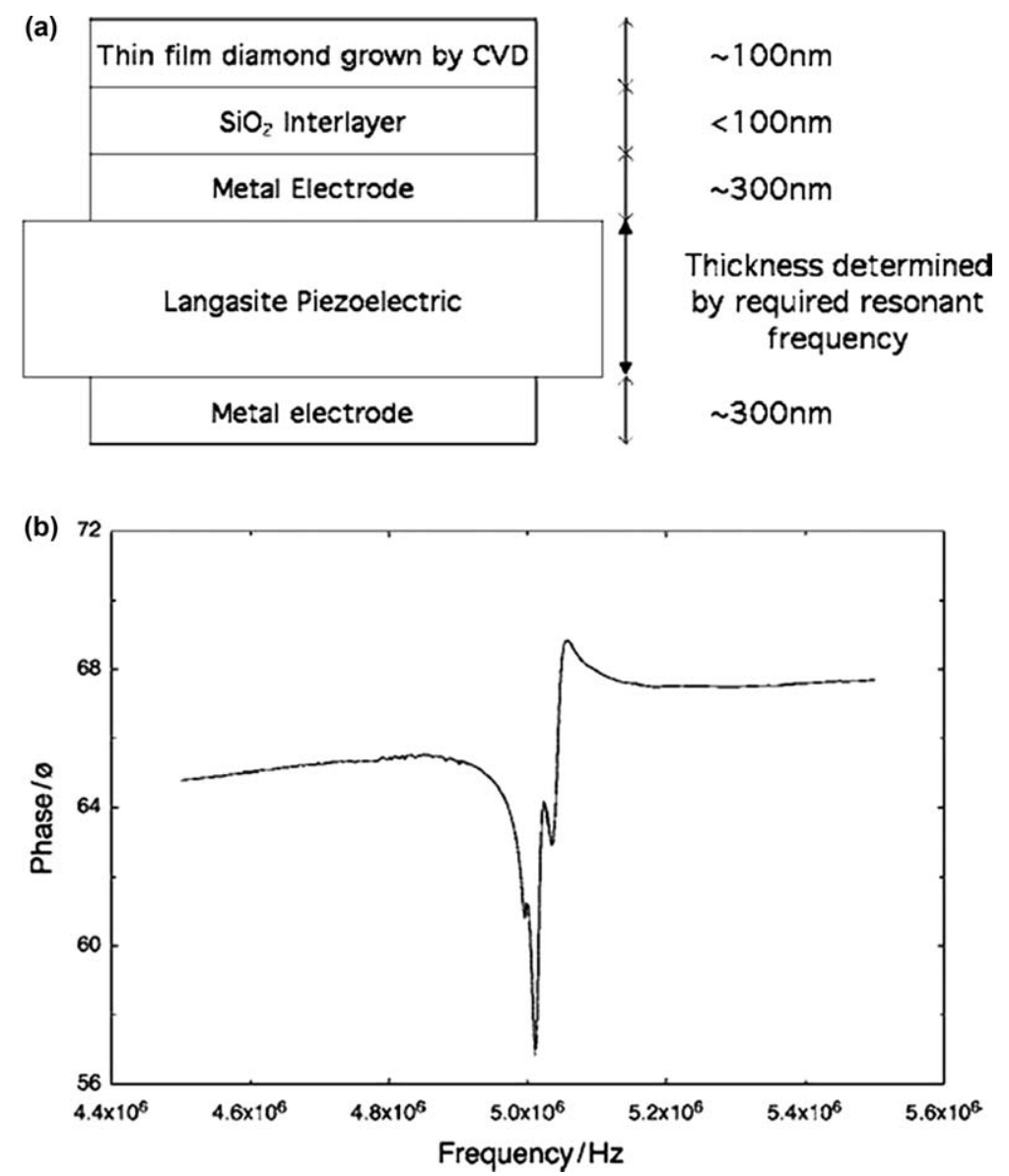

Figure 18.5 a) Schematic of the nanocrystalline diamond-coated langasite thickness shear mode resonator; b) phase shift plotted against frequency, showing the resonance of the nanocrystalline diamond-coated langasite thickness shear mode resonator.

(Reprinted with permission from ref. 39, American Institute of Physics.) 


\subsection{Diamond Cantilevers}

Cantilevers were originally developed in the 1980s as tips for scanning probe microscopy. Since then, extensive research efforts has been dedicated to the fabrication and characterisation of microscale cantilevers useful for atomic force microscopy (AFM) probes. As a result, more advanced probes have become available; but when combined with research work from other areas of science, these efforts have also led to the development of new families of mechanical sensors, including, for instance, accelerometers and also chemical and biochemical sensors. Thus, the feasibility to detect specific gases, enzymes, proteins or DNA sequences using accurate mass-sensitive micro-cantilevers was demonstrated. ${ }^{40}$ In contrast with most other gravimetric transducers, cantilevers are not made of, nor do they depend on, piezoelectric materials; although, in some instances, piezoelectric gauges have been integrated to the transducer as part of the resonance frequency readout system. While MEMS can take many different shapes, devices with very simple rectangular beam configurations appear highly suitable for transducing physical, chemical or biological stimuli into useful electrical signals. AFM probes are generally fabricated from a highly inert material, such as silicon nitride but, for chemical sensing applications, a wide range of materials have been tested. Amongst those, diamond offers probably the best performance in terms of mechanical properties and chemical inertness. Micro-electro-mechanical systems (MEMS) and, more precisely, cantilevers are relatively recent technologies when compared to more conventional transducer technologies, like optical or electrochemical methods. Nevertheless, they are recognised as promising sensing devices for chemical and biological analysis because they can offer low instrumental detection limits along with high miniaturisation and integration capabilities.

\subsubsection{Transduction Principles}

Cantilevers may operate either in the static mode or in the dynamic mode. In the static mode the surface energy changes due to chemical interactions occuring at one surface of the device according to Stoney's law. ${ }^{2}$ This results in a bending of the cantilever that is often detected optically. In this case the transduction mechanism is based on asymmetrical changes in the surface stress and there is no mass measurement involved. The sensitivity of the device increases with decreasing stiffness of the cantilever materials. Therefore, diamond is clearly not suitable for this mode of transducer operation and polymer-based cantilevers are preferred.

In the resonant regime the transduction mechanism is rather complex and is still subject to debate. Rectangular cantilevers operating in vacuum or in gases can be considered as weakly damped mechanical oscillators, ${ }^{40}$ for which the spring constant $k$ can be approximated as:

$$
k=\frac{E t^{3} w}{4 l^{3}}
$$


where $E$ is the Young's modulus of the material composing the cantilever, and $w, t$ and $l$ are the width, thickness and length of the cantilever, respectively. In this regime the cantilever resonance frequency extracted from Euler-Bernoulli beam theory is given by:

$$
f_{0}=\frac{1}{2 \pi} \sqrt{\frac{k}{m_{0}}},
$$

where $m_{0}$ is the effective mass of the cantilever. In such low damping media the cantilever response may be affected both by gravimetric loading on the cantilever and spring constant variation as a result of chemical and physical interactions occurring at the surface of the transducer. Thus, the relative cantilever resonance frequency change due to a variation of mass $(\Delta m)$ and stiffness $(\Delta k)$ can be expressed by: ${ }^{41}$

$$
\frac{\Delta f}{f_{0}} \cong\left(\sqrt{\left(1+\frac{4 l^{3} \Delta k}{E w t^{3}}\right)} \times \sqrt{\left(1-\frac{\Delta m}{m}\right)}\right)-1 .
$$

Therefore, in low damping media, the cantilever is a true mass-sensitive device only if the spring constant $k$ does not change during the measurement. This is a condition that may not be satisfied, for instance, when the elasticity of the selective layer is affected by chemical exposure. According to eqn 18.2, the higher the Young's modulus, the higher the resonance frequency of the cantilever. This equation implies, for instance, that, for equivalent geometries, a rectangular diamond cantilever will typically resonate at twice the resonant frequency of the same silicon cantilever in a vacuum. Let us assume now that our two isometric cantilevers, resonating for instance at $10 \mathrm{kHz}$ for the silicon device and at $20 \mathrm{kHz}$ for the diamond device, are subject to a mass uptake of $10 \mathrm{ng}$ without affecting the spring constant of both resonators. In this case eqn 18.3 would show that the resonance frequency variation of the diamond cantilever is approximately $400 \mathrm{~Hz}$, while that of the silicon device is around $290 \mathrm{~Hz}$. Therefore, in low damping media, it is reasonable to accept that the gravimetric sensitivity of the diamond transducer is significantly higher than its silicon counterpart.

In fluids, mechanical deformations of the cantilevers involve appreciable dissipation of mechanical energy into thermal energy. This energy dissipation can be quantified using the quality factor (or Q-factor). This depends on several parameters, such as cantilever material and shape and clamping losses, as well as the viscosity of the medium. When the cantilevers are long enough, clamping loss may be neglected and the Q-factor can be calculated using the vibrating sphere model in viscous fluids. ${ }^{42}$ Here, the Q-factor is given by:

$$
\begin{gathered}
Q=\frac{\chi^{2} \sqrt{\rho E}}{12 \pi \sqrt{3}} \frac{w t^{2}}{\mu l R\left(1+\frac{R}{\delta}\right)} \\
R=\sqrt{\frac{w l}{\pi}}
\end{gathered}
$$




$$
\delta=\sqrt{\frac{\mu}{\pi \rho f_{0}}},
$$

where $\chi$ is a constant relative to the first vibration mode $(\chi=1.875), w, l$ and $t$ are the width, length and thickness of the cantilever, respectively, $E$ is Young's modulus, $\mu$ is the viscosity of the medium, $\mathrm{R}$ is the radius of the equivalent sphere, which can be approximated by eqn 18.5 and $\delta$ is the boundary layer thickness calculated from eqn 18.6, where $\rho$ is the density of the medium. In a given medium and for a given geometry, these equations imply that the Q-factor of a diamond cantilever (for $\rho=3.50 \mathrm{~g} \mathrm{~cm}^{-3}$ and $E=1000 \mathrm{GPa}$ ) is typically three times higher than a silicon counterpart (for $\rho=2.33 \mathrm{~g} \mathrm{~cm}^{-3}$ and $E=160 \mathrm{GPa}$ ). This was verified experimentally by Bongrain et al. (Figure 18.6). ${ }^{43}$ In high damping media one has to take this dissipation of mechanical energy into account when calculating the resonance frequency of the cantilevers. The later can thus be expressed as:

$$
f_{0, Q}=\frac{1}{2^{\frac{3}{2}} \pi} \sqrt{\frac{k}{m_{0}}} \frac{\sqrt{2 Q-1}}{Q} .
$$

Theoretically, this expression shows that the higher the Q-factor, the higher the resonance frequency. Hence, the resonance frequency of microscale diamond cantilevers tends also to be higher than cantilevers made of other materials in viscous media. This is a very important observation because, today, damping is a serious limitation for measurements in the resonant regime in liquids. As a result, most cantilever-based chemical or biological sensors reported in the literature are operating in the bending mode. Here, diamond holds significant potential for improving the transduction

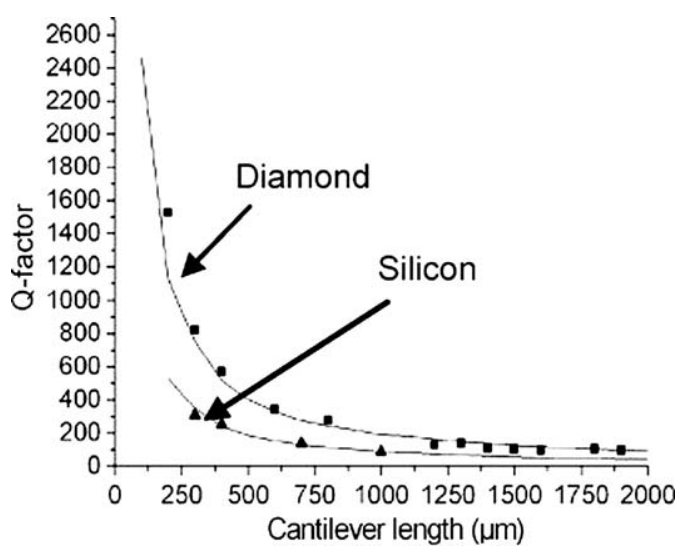

Figure 18.6 Measured and calculated Q-factor values of fabricated $4.6 \mu \mathrm{m}$ thick bare diamond cantilevers and comparison with the calculated values of identical silicon structure Q-factors; values found in the literature for equivalent silicon cantilevers.

(Reprinted from ref. 43 with permission from Elsevier.) 
performance of cantilevers in liquid environments, a prerequisite for biosensor applications.

Nevertheless, there is some strong evidence showing that chemical and physical interactions between the cantilever surface and its environment do affect the cantilever stiffness, thus making the gravimetric analysis much less straightforward. Several models were proposed to explain the cantilevers sensitivity to surface stress induced by molecular interactions based on strain-independent contributions, but they were questioned because they do not take into account stress relaxation possibilities. Moreover, the classical one-dimensional beam theory also predicts that cantilever stiffness is independent of strain-independent surface stress. Today, this still has not been elucidated as other groups have shown that three-dimensional models indicate that strain-independent surface stress indeed influences cantilever stiffness. In contrast others groups have proposed that cantilever resonance frequency surface stress is influenced by strain-dependent surface stress. The influence of this contribution upon the cantilever flexural rigidity can be described from one-dimensional beam theory by:

$$
(E I)^{b}=(E I)^{0}\left(1+3 \frac{b_{\text {top }}+b_{\text {bottom }}}{E t}\right),
$$

where $(E I)^{b}$ and $(E I)^{0}$ are the cantilever flexural rigidity with and without strain-dependent surface stress, respectively, and $b_{\text {top }}$ and $b_{\text {bottom }}$ are the strain-dependent surface stress on the top and the bottom surface of the cantilever, respectively. ${ }^{41}$ The relation between a rectangular shape cantilever stiffness coefficient $k$ and $E I$ is given by:

$$
k=\frac{3 E I}{l^{3}} .
$$

Eqns 18.1, 18.2 and 18.8 predict a weak sensitivity of cantilevers upon surface stress variations in the order of a few hundred $\mu \mathrm{Hz}$ for typical surface stress variations of several hundred $\mathrm{mN} \mathrm{m}^{-1}$ for silicon cantilevers. According to eqn 18.8, the sensitivity would be even lower for a diamond cantilever of the same size, since it is inversely proportional to the Young's modulus. Hence, according to the strain-dependent model, the advantage of using diamond structures for their superior Q-factor in liquids may be negated by a lower sensitivity. Nevertheless, the experimental values of the frequency shifts induced by biological/chemical species immobilisation on cantilever surfaces are reported in the range of several tens of $\mathrm{Hz}$ for, typically, hundreds of $\mathrm{mN} \mathrm{m}^{-1}$ surface stress variations. These later values are in closer agreement with the values calculated using strain-independent surface stress models, which are controversial in the literature. These considerations illustrate evidence of a serious mismatch existing between theoretical predictions and experimental observations. Until this is resolved, the real advantage of using diamond cantilevers in the resonant regime in liquids for their exceptional mechanical properties is still to be demonstrated. 


\subsubsection{Fabrication Methods}

Single-crystal and polycrystalline diamond cantilevers have been reported in the literature. Nevertheless, most of them fall into the second category since the advantages of single-crystal cantilevers, typically a slightly higher $\mathrm{Q}$-factor, ${ }^{44}$ does not add much value to chemical sensors, as opposed to the main drawback associated with low availability of single crystals and poor compatibility with mass production. Therefore, in the following we will concentrate only on polycrystalline diamond cantilevers.

The patterning of diamond for MEMS fabrication poses serious challenges because the chemical resilience of the material prevents the use of wet etching protocols. Despite this limitation, there is still a real interest for the use of patterning processes that remain compatible with conventional silicon and microsystem fabrication techniques. Taking this into account, two main approaches have been considered. Perhaps the most obvious one consists of selectively etching a continuous film of diamond by dry-etching techniques (Figure 18.7a). Thus, four primary diamond dry-etching methods that are compatible with classical clean room lithographic techniques have been reported over the years. ${ }^{45}$ They include electron cyclotron etching (ECR), ion bean etching (IBE), reactive ion etching (RIE) and inductively coupled plasma etching (ICP). The most frequently used gases for etching diamond using these techniques are $\mathrm{O}_{2}, \mathrm{CF}_{4}$ and $\mathrm{SF}_{6}$. These dry-etching techniques may be used either to etch monocrystalline or polycrystalline diamond.

In the case of polycrystalline diamond, another way of patterning diamond consists of selectively seeding the substrate in order to grow only in areas of interest. Several bottom-up methods of this kind have been reported that include the use of a photoresist loaded with diamond powder, the use of masks, such as $\mathrm{SiO}_{2}$ or Ti/Pt, or even by direct spray writing. ${ }^{45}$ Seeding may be performed by the deposition of diamond nanoparticles over the substrate or by bias enhanced nucleation. ${ }^{46}$ However, these methods are limited in the resolution that can be achieved and, hence, to large size cantilevers, typically of several tens of microns in length and width. Moreover, unwanted nucleation generally occurs during growth in undesired areas, which contribute also to the loss of resolution of the patterns. In order to improve the resolution of patterns, variations of the technique were developed using $\mathrm{SiO}_{2}{ }^{47}$ or silicon molds ${ }^{43,46}$ (Figure 18.7b). Here, silicon molds are preferred since $\mathrm{SiO}_{2}$ molds may be etched by the hydrogen plasma, which contribute to a loss of resolution.

\subsubsection{Diamond-based Resonant Cantilever Chemical Sensors}

Chemical or biochemical sensing with resonant cantilevers is a fairly recent approach, although several proofs of concept have already been reported in the literature in the last twenty years or so. The materials used to fabricate 
(a)

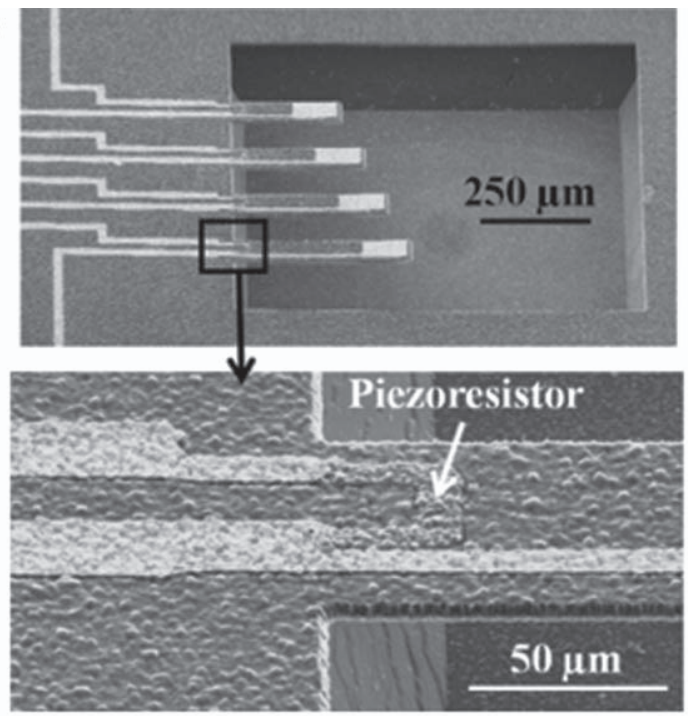

(b)

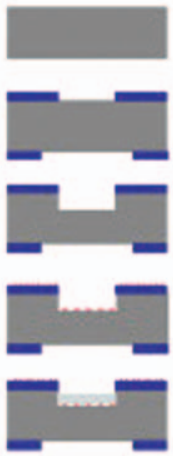

silicon substrate

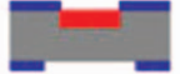

diamond growth

\section{thermal oxidation} -photolithography

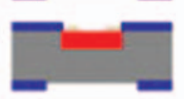

-Ti/gold layer

deposition

silicon DRIE

photolithography

diamond nano

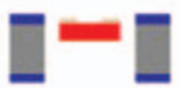

MEMS releasing

particles deposition

(nano seeding)

-aluminum deposition

-photolithography
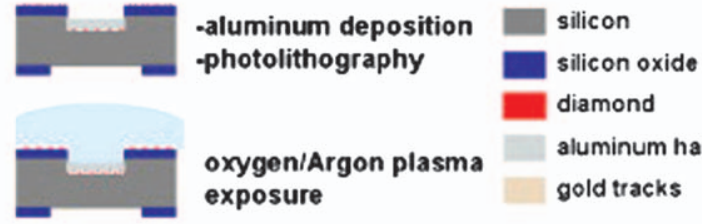

oxygen/Argon plasma

exposure

diamond

aluminum hard mask

gold tracks

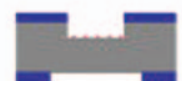

aluminum hard mask

removing

Figure 18.7 a) SEM image of the polycrystalline diamond cantilever structure used to characterise the piezoresistive effect (reprinted from ref. 45 with permission from Elsevier); b) fabrication process of diamond MEMS devices.

(Reprinted from ref. 43 with permission from Elsevier).

cantilever transducers are mostly silicon, silicon oxide or silicon nitride. Then, a selective coating is added onto one surface of the device, generally in the form of a polymer coating or chemically grafted receptors. Since the chemistry of silicon-based materials is somehow limited, a layer of gold is 
often deposited onto the transducer surface in order to allow immobilisation of receptors via thiol attachment. ${ }^{48}$ Most cantilever chemical sensors to date operate in the bending mode in liquid because the Q-factors of resonant structures in liquids are too low to perform high $\mathrm{S} / \mathrm{N}$ measurements. The use of diamond holds promise for working in the dynamic mode in liquids since its Young's modulus is sufficiently high with respect to more conventional materials, thus significantly increasing the Q-factor and enabling measurements in liquids. Moreover, the carbon surface of diamond can be used directly to attach, covalently, chemical or bio-receptors onto the transducer without the addition of a gold layer. It was shown, for instance, that the addition of a gold layer onto a diamond cantilever surface affects significantly its Q-factor. ${ }^{43}$ Diamond may also be doped with boron in order to integrate either BDD electrodes onto the structure, which can be used for localised electro-grafting on the surface, ${ }^{49}$ or piezoresistive gauges as a frequency readout strategy. ${ }^{45}$ Therefore, in principle, it is possible to fabricate single-material diamond cantilevers that can include all the necessary functions for making chemical sensors without significantly affecting the mechanical properties of the cantilever. Despite all these assets, there are very few reports of diamond cantilevers for chemical sensing, probably because of the poor accessibility of diamond materials in the chemical sensor community.

Amongst the few reports of chemical sensing with diamond cantilevers, one can mention the study by Bongrain $e t$ al. on the response of a diamond cantilever to protonation/deprotonation events of an acid group immobilised on the surface of a bulk diamond cantilever (Figure 18.8). ${ }^{41}$ This experiment was set up to investigate the effects of surface stress variations, while avoiding significant mass loading on the cantilever. Frequency

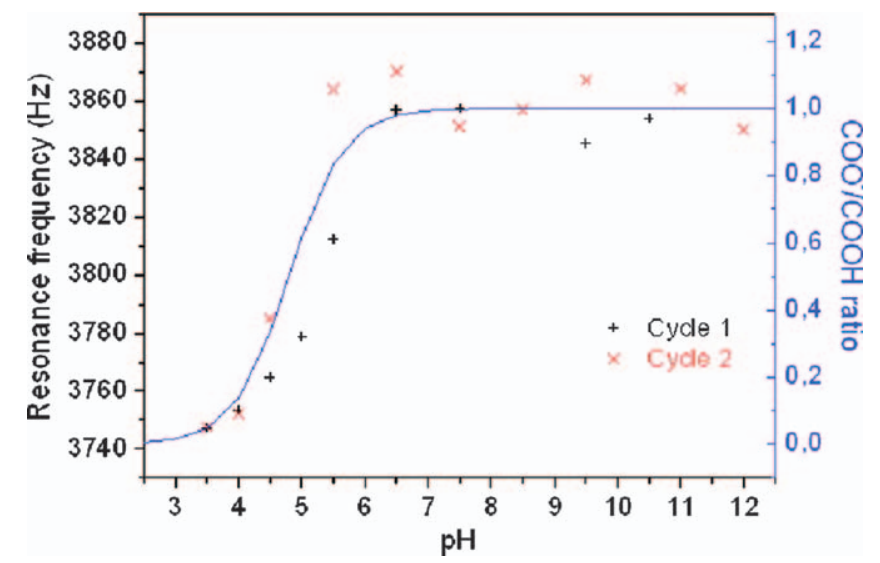

Figure 18.8 Resonance frequency variations of a diamond cantilever functionalised with carboxyl groups up $\mathrm{pH}$ cycling.

(Reprinted with permission from ref. 41. Copyright (2011) American Chemical Society.) 
variations over $100 \mathrm{~Hz}$ could be observed when cycling the $\mathrm{pH}$ from three to twelve, with a main contribution to the response arising from stiffness variations of the cantilevers due to electrostatic interactions at the surface of the sensor. Interestingly, in this experiment the carboxylic function was also immobilised covalently on the diamond surface by a one-step protocol ${ }^{27}$ comparable, in that respect, to the processes used on gold layers. This experiment confirmed that cantilevers can be extremely sensitive to chemical events occurring at their surfaces, even when abstracting the mass parameter. This effect was exploited for the detection of DNA hybridisation/denaturation events at the surface of a diamond cantilever (Figure 18.9). ${ }^{49}$ Indeed, DNA, being itself a highly charged molecule, is liable to charge interactions with
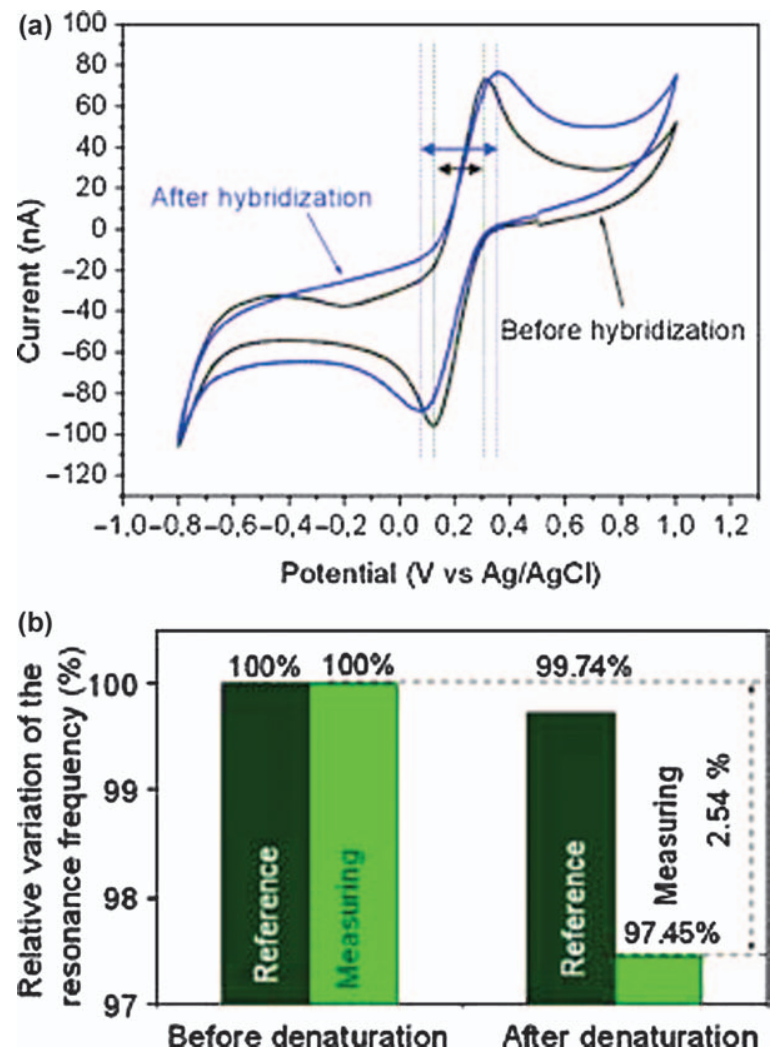

Figure 18.9 a) Cyclic voltamograms in $0.5 \mathrm{mM} \mathrm{Fe}\left(\mathrm{CN}_{6}\right)^{3-/ 4-}, 100 \mathrm{mM} \mathrm{KCl}, 100 \mathrm{mM}$ $\mathrm{KNO}_{3}$ before and after DNA hybridisation; b) resonance frequency of both reference (dark) and measuring (light) cantilevers before and after DNA denaturation in phosphate-buffered saline (PBS) solution. For the reference cantilever: $f=3121 \mathrm{~Hz}$ before, and $f=3113 \mathrm{~Hz}$ after denaturation; for the measuring cantilever: $f=2948 \mathrm{~Hz}$ before, and $f=2873 \mathrm{~Hz}$ after denaturation.

(Reprinted from ref. 49 with permission, copyright (2012) John Wiley and Sons.) 


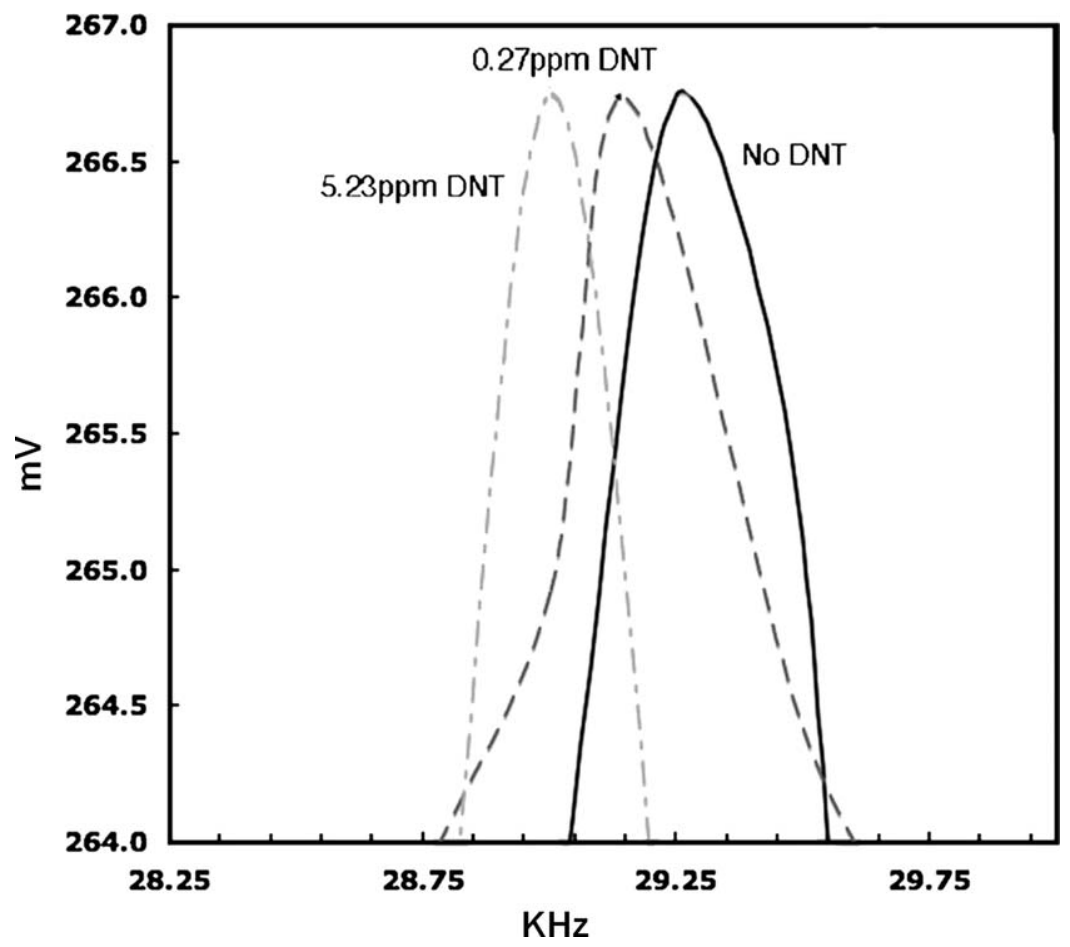

Figure 18.10 Dynamic response (expressed in $\mathrm{mV}$ ) of a nanodiamond-coated cantilever before exposure to DNT and following exposure to 0.27 and 5.23 ppm DNT vapour.

(Reprinted with permission from ref. 50, American Institute of Physics.)

neighbouring DNA molecules or ions from the surrounding medium. Probe DNA was immobilised via an electrochemical process on the surface of a diamond cantilever onto which a BDD electrode was overgrown. The possibility to detect complementary 32 base pair DNA was demonstrated.

Finally, diamond may also be used as a chemically sensitive coating deposited onto a silicon cantilever. This approach allows the use of well-established silicon cantilever technology, while benefiting from the properties of diamond surfaces for sensing applications. 2,4-dinitrotoluene (DNT), a safe analog for the detection of the explosive trinitrotoluene, was used in order to demonstrate this proof of concept. ${ }^{50} \mathrm{~A}$ respectable sensitivity of $0.77 \mathrm{~Hz} \mathrm{ppb}^{-1}$ was achieved by such a sensor (Figure 18.10).

\subsection{Surface Acoustic Wave Resonators}

\subsubsection{Generality}

Surface acoustic wave (SAW) chemical sensors constitute another family of so-called "mass sensitive sensors", although the sensing mechanisms are 
actually fairly complex and include some visco-elastic and electro-acoustic components along with the gravimetric sensitivity. ${ }^{51,52}$ There are many types of SAW sensor devices but they all rely on the same fundamental principle: the perturbation upon chemical stimuli of an acoustic wave piezoelectrically generated and travelling across the surface of the transducer. In this chapter we will focus on Rayleigh SAW (R-SAW) transducers since there are very few reports, if any, of diamond-based SAW chemical transducers based on other propagation modes. R-SAW transducers are particularly interesting for sensing in the gas phase. The most basic R-SAW structure is a two-terminal delay line transducer, as illustrated in Figure 18.11. Here, the acoustic wave is generated at the transmitter electrode. It travels along the surface of the substrate and is then transformed back into an electrical signal by the reverse piezoelectric effect at the receiving electrode. A selective layer is generally deposited on the delay line. Interactions of this layer with target chemicals induce amplitude attenuation, phase difference or frequency shift. ${ }^{2}$ Other SAW sensors employ a resonator arrangement, which allows better sensor performances, particularly because of higher Q-factors. In general, since the wave energy is confined to within one wavelength from the surface, this characteristic yields sensors that are very sensitive to chemical interactions occurring at the surface. ${ }^{51}$ As a result, the sensitivity of SAW transducers is typically one order of magnitude higher than that of QCM. ${ }^{2}$

The delay line transducer operates most efficiently when the SAW wavelength $\lambda$ matches the transducer periodicity $d$. This occurs when the

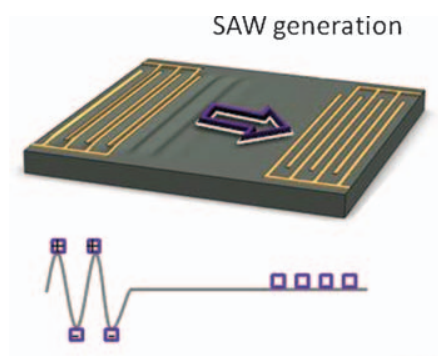

Transmitter
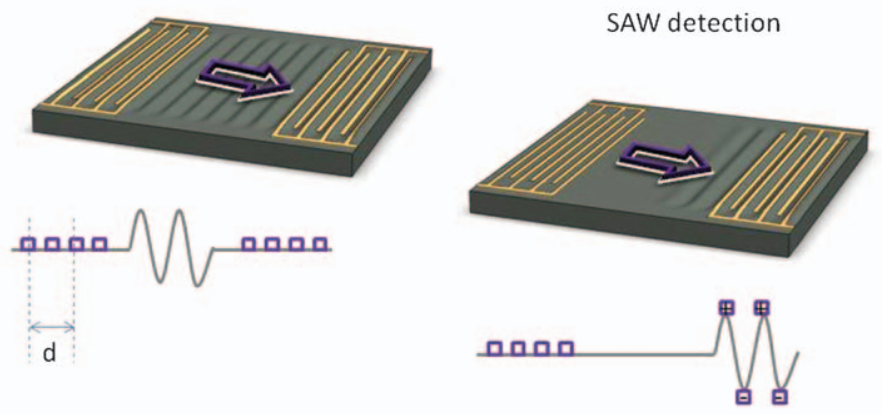

Receiver

Figure 18.11 Generation of an acoustic wave on a delay line resonator (schematic by B. Bazin). 
transducer is excited at the synchronous frequency $f_{0}$, which satisfies eqn 18.10, where $v_{\mathrm{O}}$ is the SAW propagation velocity.

$$
f_{0}=\frac{v_{\mathrm{O}}}{d}
$$

The acoustic velocity in diamond is highest (typically $20000 \mathrm{~m} \mathrm{~s}^{-1}$ in the single crystal); therefore, SAWs travel faster in diamond than in conventional piezoelectric materials. Besides, according to eqn 18.10, the higher $v_{\mathrm{O}}$, the higher the frequency. This has been the basis for the development of high frequency diamond-based resonators operating in the $\mathrm{GHz}$ range for electronic applications. Since diamond is not a piezoelectric material, typically $\mathrm{ZnO}$ or AlN layers are deposited onto the diamond to piezoelectrically generate the acoustic wave. ${ }^{53,54}$

In the case of R-SAW transducers, the effect of mass loading on the wave propagation may be expressed by:

$$
\frac{\Delta v}{v_{0}}=-C_{\mathrm{m}} f_{0} \rho_{\mathrm{s}}
$$

where $C_{\mathrm{m}}$ is a mass sensitivity factor and $\rho_{\mathrm{s}}$ the crystal density. The mass sensitivity $S_{\mathrm{m}}$ is given by:

$$
S_{\mathrm{m}}=\frac{1}{\Delta m}\left(\frac{\Delta v}{v_{0}}\right) .
$$

Thus, the sensitivity of a Y-cut quartz R-SAW delay line transducer operating at $97 \mathrm{MHz}$ is approximately $12200 \mathrm{~Hz} \mathrm{~cm}^{2} \mu \mathrm{g}^{-1} .^{51}$ Nevertheless, eqn 18.11 suggests that, for R-SAWs, the sensitivity increases with the frequency of the device. Therefore, one way to increase the mass sensitivity of a SAW transducer intuitively is to work at high frequencies. Hence, diamond SAW resonators should be good candidates for designing high sensitivity chemical sensors. In practice this is not entirely true because for high frequencies typically in the $\mathrm{GHz}$ range, the acoustic wave propagation becomes highly affected by the presence of the selective coating deposited onto the transducer surface, which degrades also the Q-factor significantly. The sensitivity to other environmental parameters is also potentially higher and so is the noise level. Hence, a compromise has to be found and, generally, SAW sensors operate in the range 100-500 MHz. At those frequencies, there is no need to use diamond as a waveguide. However, diamond has been used on such devices as a suitable sensing interface layer since it offers a highly stable versatile surface for the design of selective coatings.

\subsubsection{Diamond Nanoparticles-coated SAW Chemical Sensors}

R-SAW sensors are promising for the detection of chemicals in the gas phase due to their extremely high sensitivity. The selective coating here is generally the limiting element in terms of sensor-to-sensor repeatability, reliability 
and long-term stability. In most cases the sensitive coatings are based on polymers that are generally difficult to deposit homogeneously onto the transducer surface. ${ }^{55-57}$ Other types of coatings have also been considered, including carbon nanotubes ${ }^{58}$ or, more recently, graphene sheets, ${ }^{59}$ but they also suffer from coating difficulties. Indeed, a strict control of the thickness uniformity, viscosity and film adherence of the coating is necessary in order to obtain reliable performances as any defects present on the SAW propagation path is known to degrade the performances of the sensors. ${ }^{57}$

In this context nanoparticles of diamond have been considered as an alternative sensitive coating that could solve some of the issues encountered with other known selective coatings. Recent developments in conformal coating with diamond nanoparticles using layer-by-layer deposition methods have enabled the deposition of diamond nanoparticle thin films onto SAW transducers $^{60}$ (Figure 18.12). For example, negatively charged diamond particles were immobilised onto R-SAW resonators using a cationic polymer. ${ }^{61,62}$ Diamond nanoparticles from detonation can be found in nanometer sizes and can thus be deposited using this approach as single or multiple layers on sensors.

In addition to the high stability of the particles due to their carbon $\mathrm{sp}^{3}$ nature, thin films of diamond nanoparticles offer several advantages for gas sensing applications using R-SAW transducers. First of all, the high density of diamond prevents absorption of any molecules onto the bulk of the coating. Hence, chemical interactions occur only at the surface of the particles, thus avoiding potential poisoning of the selective layer by trapping of molecules into the material. Moreover, in contrast with polymers for instance, the lack of diffusion into the bulk of the layer may improve the sensor response times since it relies mainly on adsorption kinetics at the diamond surface. For example, Chevallier et al. reported the detection of $240 \mathrm{ppb}$ 2,4-dinitrotoluene using a selective coating based on a porphyrin

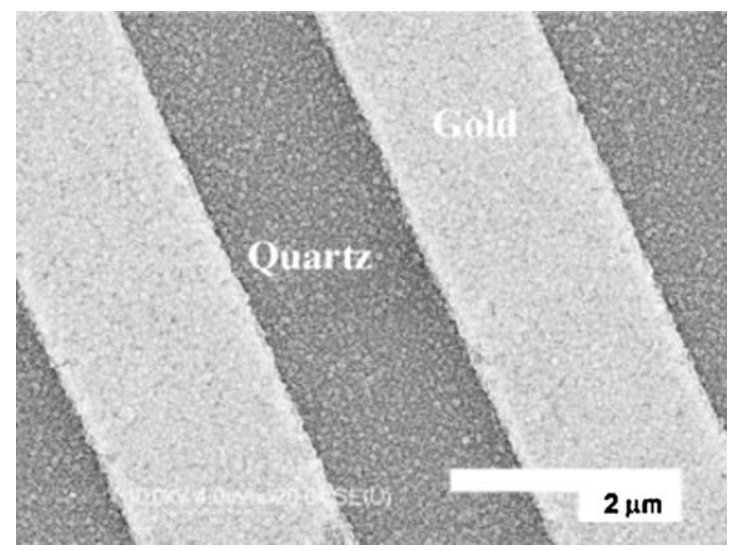

Figure 18.12 SEM image of a SAW sensor with a slight nanodiamond coating. (Reprinted from ref. 61 with permission from Elsevier.) 

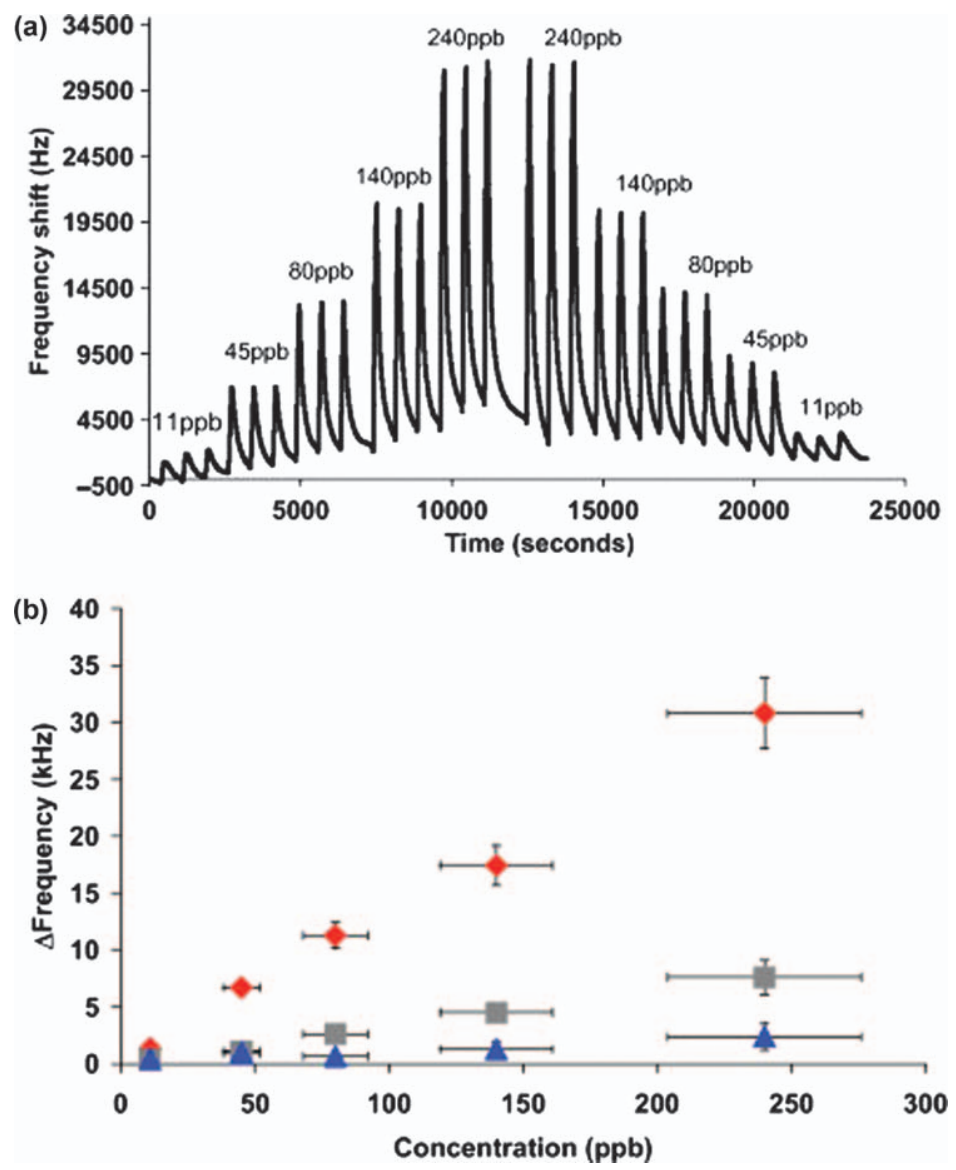

Figure 18.13 a) Transient response of SAW sensor coated with ZnTMPynanodiamond at room temperature to different concentrations of DNT; b) calibration curve obtained from four separate SAW sensors prepared with three layers of ZnTMPy-nanodiamond (red curve), three layers of nanodiamond (gray curve) and three layers of ZnTMpy (blue curve) to DNT gas at room temperature.

(Reprinted from ref. 62 with permission from Elsevier.)

complex immobilised on diamond nanoparticles with a response time $T_{90 \%}$ of typically 250 s. $^{62}$ Here, the porphyrin complex was chosen for its strong affinity with nitroaromatic compounds. ${ }^{64,65}$ The same team showed that the use of diamond nanoparticles in this case could improve the sensitivity of the sensor with respect to the same transducer coated with porphyrin alone by, typically, a factor ten (Figure 18.13), most probably because of the higher sensing surface area created by the nanoparticle coating.

As already discussed in the earlier sections, the carbon nature of diamond offers many opportunities for attachment of a wide range of chemical or biological receptors via $\mathrm{C}-\mathrm{C}$ covalent bonding. Tard and coworkers showed, 
for example, that fluoroalcohols groups $\left(-\left(\mathrm{CF}_{3}\right)_{2}-\mathrm{OH}\right)$ could be immobilised covalently on diamond nanoparticles for the detection of organophosphorous compounds. ${ }^{63}$ This work was inspired from previous work on polymers containing fluoroalcohol moieties that exhibit strong hydrogen bond acidic affinities with hydrogen bond basic organophosphorus nerve agents. ${ }^{66}$ The sensitivity to dimethyl-methylphosphonate, a simulant for sarin gas, was comparable to that of the polymer coatings (typically $750 \mathrm{~Hz}$ ppm $\mathrm{v}^{-1}$ ), with response times typically below $1 \mathrm{~min}$. The same R-SAW sensor was also tested with sarin gas, showing again similar results. Other examples of applications using R-SAW diamond-based sensors include the detection of mustard gas and phosgene, ${ }^{63}$ or ammonia. ${ }^{61}$

\subsubsection{Toward Artificial Olfaction Using Diamond-based SAW Sensors}

In many applications of chemical or biochemical sensing, selectivity is a crucial parameter. However, the only approach to make $100 \%$ selective coatings is probably through the use of bio-receptors, such as antibodies, enzymes or DNA.Those receptors are generally suitable to detect biological targets; hence, they are particularly useful for the development of sensors for medical diagnostic. For instance, the enzyme glucose oxidase is highly suitable for the specific detection of glucose. Unfortunately, the use of bioreceptors for the detection of non-metabolic species is very limited. Amongst the few examples Kalaji and co-workers developed specific proteins for the identification of explosives. ${ }^{67}$ Furthermore, bio-receptors have a limited lifetime, especially if not kept at an adequate temperature, and in suitable $\mathrm{pH}$ or buffer conditions. Hence, they are not suitable selective coatings for measurements in the gas phase. For these applications, more stable selective coatings must be developed, but chemistry generally fails to provide $100 \%$ selective coatings. One approach has been to fabricate imprints of target species using molecularly imprinted polymers, although this approach has been unconvincing so far. The other approach is to use sensor arrays in which each individual sensor features a broad selectivity to chemicals or family of chemicals. Upon exposure to vapours, the responses of different sensors in the array are analysed using a statistical multi-parametric approach, such as an artificial neural network, in order to identify the fingerprint of the target vapour. Such an approach has been known for many years as an "electronic nose". ${ }^{6}$

For such artificial olfaction applications, R-SAW sensors are highly promising because they offer a high sensitivity in the ppb range along with the possibility to address, as discussed before, any molecule, since they are mass transducers. Diamond nanoparticle coatings, as described in the previous section, appear as highly promising for such applications since they may be used as a robust generic sensing interfaces of which the chemical affinity may be tuned by various surface chemical terminations. 
This principle was demonstrated by Chevallier and co-workers by comparing the sensitivity of two diamond nanoparticle-coated R-SAWs with different surface terminations to various chemical vapours. ${ }^{61}$ In one sensor the surface of diamond was hydrogen terminated and in the other sensor the diamond surface was highly oxidized. Figure 18.14a shows the typical response of the two sensors to $30 \mathrm{ppm}$ volume ammonia vapours. Here, both sensors are giving a response; hence, neither of them is very specific. Nevertheless, the "oxidized diamond" sensor is typically ten times more sensitive than the "hydrogen-terminated diamond" sensor due to the polar affinity of the oxide groups with ammonia vapours. These two sensors were also exposed with other vapours and, again, the affinities of the two sensors to the different chemicals appeared to be clearly different (Figure 18.14b).
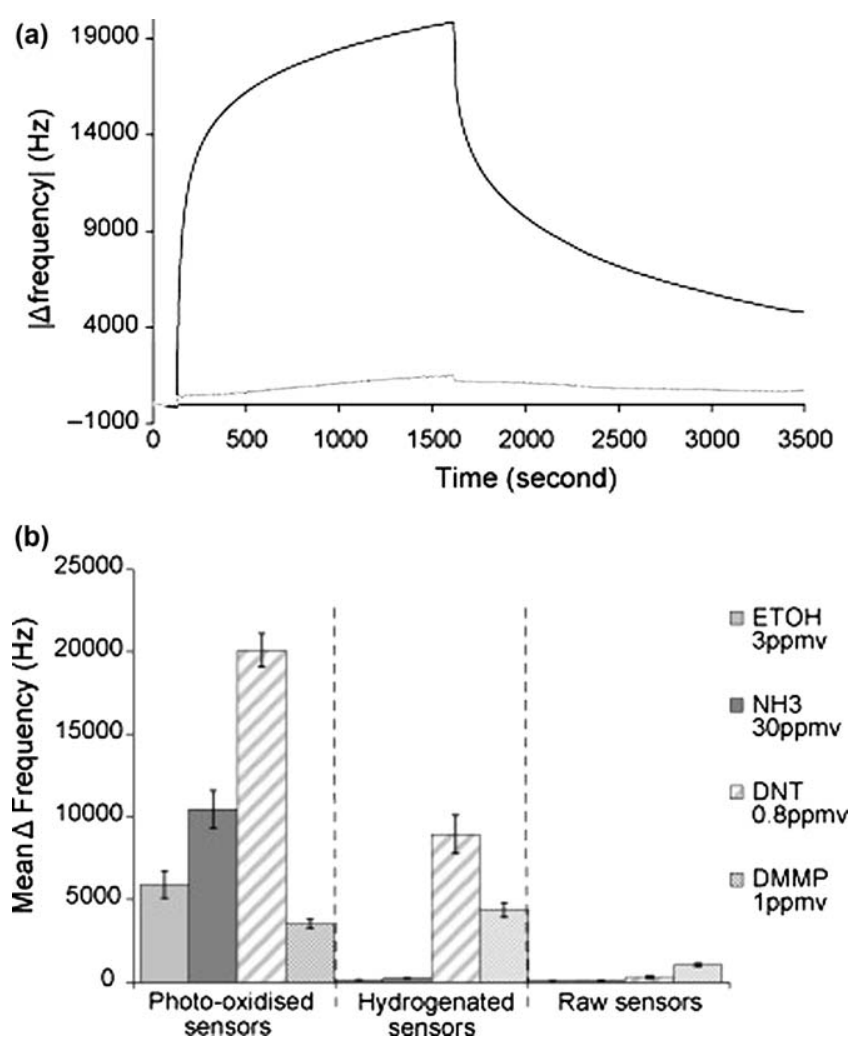

Figure 18.14 a) Response of hydrogenated nanodiamond-coated transducer (dark gray curve) and a photo-oxidised nanodiamond-coated transducer (black curve) to a $30 \mathrm{ppmv} \mathrm{NH}_{3}$ exposure; b) mean response of four hydrogenated and four photo-oxidised nanodiamond-coated transducers, respectively, to successive $60 \mathrm{~s}$ exposures to ethanol, $\mathrm{NH}_{3}$, DNT and DMMP.

(Reprinted from ref. 61 with permission from Elsevier.) 
This somewhat basic experiment demonstrated that simple surface modifications can have a drastic influence on the selectivity of the diamond based R-SAW sensors. Many wet or plasma processes have been described in the literature to modify the chemical termination of diamond surfaces, thus highlighting the opportunities of development for such sensor arrays. Research in this field is very recent and considerable further work will be necessary in order to build specific reliable sensor arrays to comply with the needs of potential users.

\subsection{Conclusions}

In summary, diamond features exceptional chemical and physical properties that can be beneficial to improve not only the robustness and reliability of chemical sensors in the field but also their sensing performances. Thus, a number of diamond-based chemical or biochemical sensors have been reported in the last twenty years, often showing highly promising performances. They include, for instance, solid-state sensors, field effect transistors and electrochemical sensors. Amongst them, diamond-based gravimetric sensors, such as SAW sensors and cantilevers, offer the possibility of high sensitivity detection of a wide range of possible analytes at potentially low costs and with a high level of miniaturisation. Research in this field is still at a fairly early stage and diamond material in its various forms, from nanoparticles to bulk single crystals, has still a lot to offer toward highly innovative new generations of sensors.

\section{References}

1. A. Hulanicki, S. Geab and F. Ingman, Pure Appl. Chem., 1991, 63, 1247.

2. J. Janata, Principles of Chemical Sensors, Springer, Verlag New York Inc., 2nd edition, 2009, p. 374.

3. J. de Sanoit, E. Vanhove, P. Mailley and P. Bergonzo, Electrochimica Acta, 2009, 544, 5688.

4. B. V. Sarada, Tata N. Rao, D. A. Tryk and A. Fujishima, Anal. Chem., 2000, $72,1632$.

5. O. Auciello, S. Pacheco, A. V. Sumant, C. Gudeman, S. Sampath, A. Datta, R. W. Carpick, V. P. Adiga, P. Zurcher, Z. Ma, H. C. Yuan, J. A. Carlisle, B. Kabius and J. Hiller, IEEE Microwave Magazine, 2008, 8, 61.

6. J. K. Luo, Y. Q. Fu, H. R. Le, J. A. Williams, S. M. Spearing and W. I. Milne, J. Micromech. Microeng., 2007, 17, S147.

7. P. Hess, J. Appl. Phys., 2012, 111, 051101.

8. S. Balachandran, T. Weller, A. Kumar, S. Jeedigunta, H. Gomez, J. Kusterer, E. Kohn, Nanocrystalline Diamond for RF-MEMS Applications, Emerging Nanotechnologies for Manufacturing, ed. W. Ahmed and M. J. Jackson, Elsevier, Oxford, UK, 2010, pp. 277-300.

9. N. Sepulveda, D. Aslam and J. P. Sullivan, Diamond Relat. Mater., 2006, 15, 398. 
10. S. Shikata, S. Fujii and T. Sharda, Diamond Relat. Mater., 2009, 18, 253.

11. Y. Gurbuz, W. P. Kang, J. L. Davidson and D. V. Kerns, Sens. Actuators B, 2004, 99, 207.

12. T. Takada, T. Fukunaga, K. Hayashi, Y. Yokota, T. Tachibana, K. Miyata and K. Kobashi, Sens. Actuator A-Phys., 2000, 82, 97.

13. Y. Isamu, K. Nobuyuki, N. Masayuki, S. Hiroshi, T. Katsunobo, Semiconductor gaz sensor, Brevet EP 0488352 A2, 1991.

14. R. K. Joshi, J. E. Weber, Q. Hu, B. Johnson, J. W. Zimmer and A. Kumar, Sens. Actuators B, 2010, 145, 527.

15. G. Swain, A. Anderson and J. Angus, MRS Bulletin, 1998, 23, 56.

16. J. A. Garrido, A. Härtl, S. Kuch, M. Stutzmann, O. A. Williams and R. B. Jackmann, Appl. Phys. Lett., 2005, 86, 073504.

17. A. Helwig, G. Müller, J. A. Garrido and M. Eickhoff, Sens. Actuators B, 2008, 133, 156.

18. A. Kromka, M. Davydova, B. Rezek, M. Vanecek, M. Stuchlik, P. Exnar and M. Kalbac, Diamond Relat. Mater., 2010, 19, 196.

19. Q. Wang, S. L. Qu, S. Y. Fu, W. J. Liu, J. J. Li and C. Z. Gu, J. Appl. Phys., 2007, 102, 103714.

20. M. Davydova, A. Kromka, B. Rezek, O. Babchenko, M. Stuchlik and K. Hruska, App. Surf. Sci., 2010, 256, 5602.

21. J.-P. Mazellier, M. Mermoux, F. Andrieu, J. Widiez, J. Dechamp, S. Saada, M. Lions, M. Hasegawa, K. Tsugawa, P. Bergonzo and O. Faynot, J. Appl. Phys., 2011, 110, 084901.

22. Y. Gurbuz, W. P. Kang, J. L. Davidson and D. V. Kerns, Sens. Actuators B, 1998, 49, 115.

23. A. Balducci, A. D’Amico, C. Di Natale, M. Marinelli, E. Milani, M. E. Morgada, G. Pucella, G. Rodriguez, A. Tucciarone and G. VeronaRinati, Sens. Actuators B, 2005, 102, 111.

24. X. Checoury, D. Neel, P. Boucaud, C. Gesset, H. Girard, S. Saada and P. Bergonzo, Appl. Phys. Lett., 2012, 101, 171115.

25. M. J. Burek, N. de Leon, B. J. Shields, B. J. M. Hausmann, Y. Chu, Q. Quan, A. S. Zibov, H. Park, M. D. Lukin and M. Loncar, Nano Lett., 2012, 12, 6084.

26. P. Bergonzo, R. B. Jackman, in Thin Film Diamond, Part II, eds C. E. Nebel, J. Ristein, vol. 77, 2003, Academic Press, San Diego, USA, p. 197.

27. C. Agnès, S. Ruffinatto, E. Delbarre, A. Roget, J.-C. Arnault, F. Omnès and P. Mailley, IOP Conf. Series: Materials Science and Engineering, 2010, 16, 012001.

28. S. Q. Lud, M. Steenackers, R. Jordan, P. Bruno, D. M. Gruen, P. Feulner, J. A. Garrido and M. Stutzmann, J. Am. Chem. Soc., 2006, 128, 16884.

29. C. E. Nebel, B. Rezek, D. Shin, H. Uetsuka and N. Yang, J. Phys. D: Appl. Phys., 2007, 40, 6443.

30. J.-H. Yang, M. Degawa, K.-S. Song, C. Wang and H. Kawarada, Mater. Lett., 2010, 64, 2321. 
31. K.-S. Song, T. Hiraki, H. Umezawa and H. Kawarada, Appl. Phys. Lett., 2007, 90, 063901.

32. E. Vanhove, J. de Sanoit, P. Mailley, M.-A. Pinault, F. Jomard and P. Bergonzo, Phys. Status Solidi A, 2007, 206, 2063.

33. R. Kiran, E. Scorsone, J. de Sanoit, J.-C. Arnault, P. Mailley and P. Bergonzo, J. Electrochem. Soc., 2013, 160, H67.

34. J. Lee and S.-M. Park, Anal. Chim. Acta, 2005, 545, 27.

35. S. Szunerits, J. Niedziołka-Jönsson, R. Boukherroub, P. Woisel, J.-S. Baumann and A. Siriwardena, Anal. Chem., 2010, 82, 8203.

36. J. Weng, J. Zhang, H. Li, L. Sun, C. Lin and Q. Zhang, Anal. Chem., 2008, 80, 7075 .

37. T. A. Ivandini, D. Yamada, T. Watanabe, H. Matsuura, N. Nakano, A. Fujishima and Y. Einaga, J. Electroanal. Chem., 2010, 645, 58.

38. Y. Zhang, S. Asahina, S. Yoshihara and T. Shirakashi, J. Electrochem. Soc., 2002, 149, H179.

39. O. A. Williams, V. Mortet, M. Daenen and K. Haenen, Appl. Phys. Lett., 2007, 90, 063514.

40. N. V. Lavrik, M. J. Sepaniak and P. G. Datskos, Rev. Sci. Instrum., 2004, $75,2229$.

41. A. Bongrain, C. Agnès, L. Rousseau, E. Scorsone, J-C Arnault, S. Ruffinatto, F. Omnès, P. Mailley, G. Lissorgues and P. Bergonzo, Langmuir, 2011, 27, 12226.

42. K. Naeli and O. Brand, J. Appl. Phys., 2009, 105, 014908.

43. A. Bongrain, E. Scorsone, L. Rousseau, G. Lissorgues and P. Bergonzo, Sens. Actuators B, 2011, 154, 142.

44. M. Liao, C. Li, S. Hishita and Y. Koide, J. Micromech. Microeng., 2010, 20, 085002.

45. Z. Cao and D. Aslam, Diamond Relat. Mater., 2010, 19, 1263.

46. A. Bongrain, E. Scorsone, L. Rousseau, G. Lissorgues, C Gesset, S Saada and P Bergonzo, J. Micromech. Microeng., 2009, 19, 074015.

47. G. M. R. Sirineni, H. A. Naseem, A. P. Malshe and W. D. Brown, Diamond Relat. Mater., 1997, 6, 952.

48. M. Álvarez, L. G. Carrascosa, M. Moreno, A. Calle, A. Zaballos, L. M. Lechuga, C. Martínez and J. Tamayo, Langmuir, 2004, 20, 9663.

49. A. Bongrain, H. Uetsuka, L. Rousseau, L. Valbin, S. Saada, C. Gesset, E. Scorsone, G. Lissorgues and P. Bergonzo, Phys. Status Solidi A, 2010, 207, 2078.

50. R. K. Ahmad, A. C. Parada, S. Hudziak, A. Chaudhary and R. B. Jackman, Appl. Phys. Lett., 2010, 97, 093103.

51. D. S. Ballantine, R. M. White, S. J. Martin, A. J. Ricco, E. T. Zellers, C. G. C. Frye and H. Wohltjen, Acoustic Waves Sensors, Theory, Design, and Physico-chemical Applications, Academic Press, 1st edition, San Diego, USA, 1996, 436.

52. E. A. Ash, G. W. Farnell, H. M. Gerard, A. A. Oliner, A. J. Slobodnik, JR. and H. I. Smith, Acoustic surface waves, Springer, Verlag Berlin and Heidelberg GmbH \& Co. K, 1978, p. 331. 
53. J. G. Rodriguez-Madrid, G. F. Iriarte, D. Araujo, M. P. Villar, O. A. Williams, W. Müller-Sebert and F. Calle, Mater. Lett., 2012, 66, 339.

54. W.-C. Shih and R.-C. Huang, Vacuum, 2008, 83, 675.

55. G. Harsanyi, Polymer Films in Sensor Applications: Technology, Materials, Devices and Their Characteristics, Technomic Publishing Co., Lancaster, Basel, 1995, p. 435.

56. J. W. Grate, Chem. Rev., 2000, 100, 2627.

57. J. W. Grate and R. A. McGill, Anal. Chem., 1995, 67, 4015.

58. M. Penza, P. Aversa, G. Cassano, W. Wlodarski and K. Kalantar-Zadeh, Sens. Actuators B, 2007, 127, 168.

59. R. Arsat, M. Breedon, M. Shafiei, P. G. Spizziri, S. Gilje, R. B. Kaner, K. Kalantarzadeh and W. Wlodarski, Chem. Phys. Lett., 2009, 467, 344.

60. H. A. Girard, E. Scorsone, S. Saada, C. Gesset, J. C. Arnault, S. Perruchas, L. Rousseau, S. David, V. Pichot, D. Spitzer and P. Bergonzo, Diamond Relat. Mater., 2012, 23, 83.

61. E. Chevallier, E. Scorsone and P. Bergonzo, Sens. Actuators B, 2011, 154, 238.

62. E. Chevallier, E. Scorsone, H. A. Girard, V. pichot, D. Spitzer and P. Bergonzo, Sens. Actuators B, 2010, 151, 191.

63. B. Tard, A. Trouvé, E. Scorsone, A. Voigt, M. Rapp and P. Bergonzo, The 14th International Meeting on Chemical Sensors, 2012, 481.

64. S. Tao, G. Li and H. Zhu, J. Mater. Chem., 2006, 16, 4521.

65. S. Tao, J. Yin and G. Li, J. Mater. Chem., 2008, 18, 4872.

66. Q. Zheng, Y.-C. Fu and J.-Q. Xu, Procedia Eng., 2010, 7, 179.

67. C. D. Gwenin, M. Kalaji, P. A. Williams and R. M. Jones, Biosens. Bioelectron., 2007, 22, 2869.

68. J. Gardner, P. N. Bartlett, Sensors and Sensory Systems for an Electronic Nose, Springer, Dordrecht, Netherlands, 1992, p. 340. 